\title{
Spectral-like Duality for Distributive Hilbert Algebras with Infimum
}

\author{
Sergio A. Celani ${ }^{1}$, María Esteban² ${ }^{2}$ and Ramón Jansana ${ }^{3}$ \\ 1 CONICET and Universidad Nacional del Centro, Argentina \\ scelani@exa.unicen.edu.ar \\ 2 Universitat de Barcelona, Spain \\ mariaesteban@ub.edu \\ 3 Universitat de Barcelona, Spain \\ jansana@ub.edu
}

\begin{abstract}
We present the results of our research on stone-type dualities for certain classes of ordered algebras that do not fall within the scope of extended Priestley-duality. In a forthcoming paper 5] we study a new spectral-like duality for the class of distributive Hilbert algebras with infimum. We explain the main facts of that duality and we outline how the same strategy could be used for getting a Priestleystyle duality for the same class of algebras, as well as dualities for other classes of algebras.

Stone's duality for Boolean algebras 15 has been generalized to distributive lattices in at least three different directions (cf. 1] and its references). The approach initiated by Stone himself 15$]$ leads to a representation in terms of compactly-based sober spaces in which the collection of compact open sets is closed under finite intersections. Further generalizations of this approach lead to dualities for distributive meet-semilattices with top element [4, 12, implicative semilattices [3], Hilbert algebras ${ }^{2}$ [8] and Hilbert algebras with supremum [6]. What they all have in common is that they provide representations in terms of compactlybased sober spaces. We refer to this sort of dualities as spectral-like dualities.

A different approach initiated by Priestley 14] leads to a representation in terms of ordered Hausdorff topological spaces. We refer to this sort of dualities as Priestley-style dualities. Although both approaches have been followed to generalize the pioneering work of Jónsson and Tarski on representation of Boolean algebras with operators [13, the later has been held to be advantageous, especially considering recent developments of the theory of canonical extensions (see 11] and his references). The key point of this theory, usually called extended Priestleyduality is that the additional $n$-ary operations either preserve joins (meets) in each coordinate, or send joins (meets) in each coordinate to meets (joins). Such $n$-ary operations (called (dual) quasi-operators) are dually represented by $n+1$-ary relations satisfying certain rather natural conditions.

We focus our attention on classes of algebras that are not within the scope of extended Priestley-duality, and we provide representation theorems and spectral-like dualities for them. More precisely, we consider algebras for which a spectral-like duality has been studied, and we augment them with additional operations that are not (dual) quasi-operators. The largest class of algebras to which our study can be applied is distributive semilattices with an additional Hilbert-implication that defines the same order than the meet operation but is not necessarily its right residual. We call these algebras distributive Hilbert algebras with infimum (or $\mathbb{D H}^{\wedge}$ algebras):
\end{abstract}

\footnotetext{
${ }^{1}$ Recall that implicative semilattices are the algebraic counterpart of the implicative-conjunctive fragment of intuitionistic logic.

${ }^{2}$ Recall that Hilbert algebras are the algebraic counterpart of the implicative fragment of intuitionistic logic.
} 
Definition 0.1. An algebra $\mathbf{A}=\langle A, \rightarrow, \wedge, 1\rangle$ of type $(2,2,0)$ is a distributive Hilbert algebra with infimum or $\mathbb{D H}^{\wedge}$-algebra if:

(1) $\langle A, \rightarrow, 1\rangle$ is a Hilbert algebra, i. e. for all $a, b, c \in A$ :

(i) $a \rightarrow(b \rightarrow a)=1$,

(ii) $(a \rightarrow(b \rightarrow c)) \rightarrow((a \rightarrow b) \rightarrow(a \rightarrow c))=1$,

(iii) $a \rightarrow b=1=b \rightarrow a$ implies $a=b$.

(2) $\langle A, \wedge, 1\rangle$ is a distributive meet-semilattice with top element, i. e.

(i) the binary operation $\wedge$ is idempotent, commutative, associative,

(ii) for all $a \in A, a \wedge 1=1$,

(iii) for each $a, b_{1}, b_{2} \in M$ with $b_{1} \wedge b_{2} \leq a$, there exist $c_{1}, c_{2} \in M$ such that $b_{1} \leq c_{1}, b_{2} \leq c_{2}$ and $a=c_{1} \wedge c_{2}$.

(3) for all $a, b \in A, a \rightarrow b=1$ iff $a \wedge b=a$.

Implicative semilattices are a subclass of $\mathbb{D H}^{\wedge}$-algebras, more precisely, they are the ones for which the implication is the right residual of the meet. Under this assumption, the implication preserves meets in the second coordinate. The lack of residuation in $\mathbb{D H}^{\wedge}$-algebras implies that meets do not need to be preserved in the second coordinate, and this fact is precisely what forces us to search a completely different route for a topological representation of this class of algebras. Our representation is supported in already existing dualities for distributive semilattices and Hilbert algebras.

A basic tool for our duality are implicative filters (also called deductive systems by some authors). Implicative filters are upsets closed under implication, i.e. subsets $F$ of the algebra such that whenever $a$ and $a \rightarrow b$ are in $F$, then also $b$ is in $F$. It is well known that the lattice of implicative filters of a Hilbert algebra is distributive, then so is the lattice of implicative filters of a $\mathbb{D H}^{\wedge}$-algebra. Meet irreducible elements of this lattice give us the points of the dual space of a $\mathbb{D H}^{\wedge}$-algebra. We call these elements irreducible implicative filters. Another important tool for our duality are meet filters, i.e. upsets closed under meet. As the underlying meet semilattice is distributive, then so is the lattice of meet filters of any $\mathbb{D H}^{\wedge}$-algebra. We focus again in its meet irreducible elements, that we call irreducible meet filters.

For $\mathbb{D H}^{\wedge}$-algebras, any irreducible meet filter is an irreducible implicative filters, and this is the essential fact that allows us to represent $\mathbb{D H}^{\wedge}$-algebras by means of their irreducible implicative filters. All the concepts so far introduced are also defined for the larger class of algebras consisting of meet-semilattices (not necessarily distributive) augmented with a Hilbertimplication (called $\mathbb{H}^{\wedge}$-algebras). We may represent $\mathbb{H}^{\wedge}$-algebras following the same strategy, but we do not get a similar duality for this class, since when distributivity does not hold it may fail that irreducible meet filters are included in irreducible implicative filters. Notice that for implicative filters, both concepts of implicative filter and meet filter coincide, and this implies that the duality is substantially simplified.

Regarding the duality for objects, the strategy consists of looking at the meet operation as an additional operation on the Hilbert algebra, instead of what is customary, namely looking at the implication as an additional operation on the (semi)-lattice structure. Accordingly, the meet is represented by a subset satisfying certain conditions. Dual spaces of $\mathbb{D H}^{\wedge}$-algebras are structures of the form $\left\langle X, \hat{X}, \tau_{\kappa}\right\rangle$, where $\left\langle X, \tau_{\kappa}\right\rangle$ is the dual space of the Hilbert algebra reduct, and $\hat{X}$ is a subset of $X$ that generates a sober subspace and satisfies three extra conditions. We call these spaces $\mathbb{D H}^{\wedge}$-Spectral spaces:

Definition 0.2. A $\mathbb{D} \mathbb{H}^{\wedge}$-Spectral space is a structure $\mathfrak{X}=\left\langle X, \hat{X}, \tau_{\kappa}\right\rangle$ such that: 
1. $\left\langle X, \tau_{\kappa}\right\rangle$ is an $H$-space (cf. defn 3.4 in $[8]$ ), i. e.

(H1) $\kappa$ is a basis of open and compact subsets for $X$,

(H2) for every $U, V \in \kappa, \operatorname{sat}\left(U \cap V^{c}\right) \in \kappa$,

(H3) $\left\langle X, \tau_{\kappa}\right\rangle$ is sober.

2. $\hat{X} \subseteq X$ generates a sober subspace,

3. and for every $U, V \in \kappa$ and non-empty $\mathcal{W} \subseteq \kappa$ :

$\left(D H^{\wedge} 1\right) U^{c}=\operatorname{cl}\left(U^{c} \cap \hat{X}\right)$, $\left(D H^{\wedge} 2\right) \operatorname{cl}\left(U^{c} \cap V^{c} \cap \hat{X}\right)^{c} \in \kappa$,

$\left(D H^{\wedge} 3\right)$ if $\operatorname{cl}\left(\cap\left\{W^{c}: W \in \mathcal{W}\right\} \cap \hat{X}\right) \subseteq U^{c}$, then there are $W_{0}, \ldots, W_{n} \in \mathcal{W}$, for some $n \in \omega$, such that $\operatorname{cl}\left(W_{0}^{c} \cap \cdots \cap W_{n}^{c} \cap \hat{X}\right) \subseteq U^{c}$.

We show that spectral-duals of implicative semilattices (intorduced in [3]) can be seen as $\mathbb{D H}^{\wedge}$-Spectral spaces where $\hat{X}=X$. With respect to morphisms, duals of algebraic homomorphisms between $\mathbb{D H}^{\wedge}$ algebras are binary relations that are duals of homomorphisms between Hilbert algebras and satisfy an extra condition. We call these morphisms $\mathbb{D H}^{\wedge}$ Spectral morphisms:

Definition 0.3. A relation $R \subseteq X_{1} \times X_{2}$ is a $D H^{\wedge}$-Spectral morphism between $D H^{\wedge}$-Spectral spaces $\left\langle X_{1}, \hat{X}_{1}, \tau_{\kappa_{1}}\right\rangle$ and $\left\langle X_{2}, \hat{X}_{2}, \tau_{\kappa_{2}}\right\rangle$ if:

1. $R$ is an $H$-relation between $H$-spaces $\left\langle X_{1}, \tau_{\kappa_{1}}\right\rangle$ and $\left\langle X_{2}, \tau_{\kappa_{2}}\right\rangle$ (cf. defn 3.2 in 8 ), i. e.

(HR1) $R^{-1}(U) \in \kappa_{1}$, for every $U \in \kappa_{2}$,

(HR2) $R(x)$ is a closed subset of $X_{2}$, for all $x \in X_{1}$.

2. and for every $x \in \hat{X}_{1}$ :

$\left(D H^{\wedge} \mathrm{M}\right) \quad R(x)=\operatorname{cl}\left(R(x) \cap \hat{X}_{2}\right)$.

We show that these correspondences between objects and morphisms give us a dual equivalence of categories, and we show how the functors and natural isomorphisms involved on it are defined.

Finally we apply the duality to provide a topological characterization of several classes of filters. We dually characterize the filters involved in the duality: (irreducible) implicative filters and (irreducible) meet filters. We characterize also absorbent filters, that are the logic filters related with the assertional logic of $\mathbb{H}^{\wedge}$-algebras. We characterize optimal implicative filters and optimal meet filters as well, that are the filters involved in Priestley-style dualities for distributive semilattices and Hilbert algebras respectively (see 2,9$]$ ).

Instead of representing the meet operation by a subset, we could have chosen to represent it by a ternary relation, as we will explain. Moreover, we will outline how the same strategy could be followed for a Priestley-style duality for $\mathbb{D H}^{\wedge}$-algebras. Although $\mathbb{D H}^{\wedge}$-algebras are not even a variety, we can naturally and straightforwardly extend our results to the variety of distributive lattices extended with a Hilbert-implication (called in 10 distributive Hilbert algebras), a class of algebras for which a Priestley-style duality has been already studied in 7 ]. This variety includes Heyting algebras, and it is easy to see that extended Priestley duality can not be applied in this case, since the implication reverses joins in the first coordinate, but it does not preserve nor reverse meets nor joins in the second coordinate. The strategy again consists of looking at the lattice operations as the additional operations, and representing them by a subset. 


\section{References}

[1] G. Bezhanishvili, N. Bezhanishvili, D. Gabelaia, and A. Kurz. Bitopological duality for distributive lattices and heyting algebras. Mathematical Structures in Computer Science, 20(03):359-393, 2010.

[2] G. Bezhanishvili and R. Jansana. Priestley style duality for distributive meet-semilattices. Studia Logica, 98:83-123, 2011.

[3] S. Celani. A note on homomorphisms of Hilbert algebras. International Journal of Mathematics and Mathematical Sciences, 29(1):55-61, 2002.

[4] S. Celani. Topological representation of distributive semilattices. Scientiae Mathematicae Japonicae, 8:41-51, 2003.

[5] S. Celani and M. Esteban. Spectral-like duality for distributive Hilbert algebras with infimum. to appear.

[6] S. Celani and D. Montangie. Hilbert algebras with supremum. Algebra Universalis, 67:237-255, 2012. submitted to Algebra Universalis.

[7] S. A. Celani and L. M. Cabrer. Hilbert implications over bounded distributive lattices. manuscript, 2012.

[8] S. A. Celani, L. M. Cabrer, and D. Montangie. Representation and duality for Hilbert algebras. Cent. Eur. J. Math., 7(3):463-478, 2009.

[9] S. A. Celani and R. Jansana. On the free implicative semilattice extension of a Hilbert algebra. Math. Log. Quart., pages 1-20, 2012.

[10] A. V. Figallo, G. Ramón, and S. Saad. A note on the distributive Hilbert algebras. In Actas del V Congreso de Matemática Dr. Antonio A. R. Monteiro., pages 139-152, Bahía Blanca. Argentina, 1999. Universidad Nacional del Sur.

[11] M. Gehrke and H. A. Priestley. Duality for double quasioperator algebras via their canonical extensions. Studia Logica, 68(1):31-68, 2007.

[12] G. Grätzer. General lattice theory. Academic Press. Birkhäuser Verlag, 1978.

[13] B. Jónsson and A. Tarski. Boolean algebras with operators. I. Amer. J. Math., 73:891-939, 1951.

[14] H. A. Priestley. Representation of distributive lattices by means of ordered Stone spaces. Bull. London Math. Soc., 2:186-190, 1970.

[15] M. H. Stone. The theory of representations for Boolean algebras. Transactions of the American Mathematical Society, 39:37-111, 1936. 